\title{
COMPLETE SETS AND COMPLETION OF SETS IN BANACH SPACES
}

\author{
HORST MARTINI, PIER LUIGI PAPINI, AND MARGARITA SPIROVA
}

\begin{abstract}
In this paper we study properties of complete sets and of completions of sets in Banach spaces. We consider the family of completions of a given set and its size; we also study in detail the relationships concerning diameters, radii, and centers. The results are illustrated by several examples.
\end{abstract}

Keywords: Banach space, centers, complete set, (unique) completion, diameter, diametrically maximal set, norm, normed space, radius, self-radius

MSC (2000): 46B20, 46B99, 52A05, 52A20, 52A 21

\section{INTRODUCTION}

The notion of diametrically maximal, or complete, set is around one century old. A set is complete if all its proper supersets have a larger diameter. A few decades ago the study of these sets, initially limited to finite dimensional spaces, was extended to Banach spaces of any dimension; one of the pioneering papers concerning this extension was [1]. (Note that this paper is not so easily accessible and contains a few misprints.) New interest in these sets arose in the last few years. Among the recent papers on the subject are [10, 12, 9, 11, 4]; see also the references contained in these papers. More precisely, many recent papers deal with questions like the following ones: Given a closed, bounded and convex set $D$, which is the class of its completions? (Note that complete sets containing $D$ and having the same diameter as $D$ are called completions of $D$.) When does the class of complete sets contain it? When does such a class consist of a singleton? And in addition, are there good, or special completions?

Here we deal with these questions in a general Banach space (which might be infinite dimensional). We single out and study some simple properties, trying to give a complete picture concerning the different situations which are possible.

In Sections 2 and 3, the sizes of completions for a given set $D$ are studied, with respect to the diameter and the radius of $D$. Finally, in Section 4 we study some minimality conditions for sets concerning completions.

Let $X$ be a real Banach space (in finite dimensions also called normed or Minkowski space). We denote by $O$ the origin. For $x \in X$ and $r \geq 0, B(x, r)=\{y \in X:\|x-y\| \leq r\}$ denotes the ball with center $x$ and radius $r$.

Let $D$ be a bounded, closed and convex set containing at least two points. In the following we shall always consider sets satisfying this condition. By $\delta(D)$ we will denote the diameter of $D$, and by $\partial(D)$ its boundary.

We say that $D$ is complete or diametrically maximal - (DM) for short - if $\delta(D \cup\{x\})>\delta(D)$ for every $x \notin D$.

A completion of $D$ is a $(\mathrm{DM})$ set $D_{m}$ containing $D$ and such that $\delta(D)=\delta\left(D_{m}\right)$.

We shall also use the following notations (see [1]):

$D^{\prime}=\bigcap_{x \in D}\{B(x, \delta(D))\}$, called the ball intersection of $D$, and

$D^{c}=\bigcap_{x \in X}\{B(x, \delta(D)): D \subset B(x, \delta(D))\}$, called the ball hull of $D$. 
We have always $D \subset D^{c} \subset D^{\prime}$. Moreover (see [1, Propositions 3.1 and 3.2]), we have $\delta\left(D^{c}\right)=$ $\delta(D) ;\left(D^{c}\right)^{\prime}=D^{\prime} ; D$ is complete $\Leftrightarrow D=D^{\prime}$. Also we have $\left(D^{c}\right)^{c}=D^{c}$ (a misprint occurs in [1, Proposition 3.2]).

We recall (see, for example, [2, p. 311]) the known property

$\left(P_{1}\right) \quad D^{c}=\bigcap_{x \in D^{\prime}}\{B(x, \delta(D))\}$.

We always have (see, for example, [9, Proposition 2]; or also [13, Theorem 3]):

(1) $D^{c}=\bigcap\{A: A$ is a completion of $D\}$;

(2) $D^{\prime}=\bigcup\{A: A$ is a completion of $D\}$.

Thus $D$ has a unique completion if and only if its completion is $D^{c}=D^{\prime}$; this is equivalent to the following equalities (see [1, Theorem 3.7] or [9, Corollary 3]):

$\left(2^{\prime}\right) \quad D^{c}$ is $(D M) \Longleftrightarrow D^{\prime}$ is $(D M) \Longleftrightarrow \delta(D)=\delta\left(D^{\prime}\right)$.

We recall that for normed planes and spaces these sets have been considered in many papers: from the not so recent paper [2], to the recent ones [12, 7], 8], and [9], whose results partly overlap with some results in [1].

\section{ON THE COMPLETIONS OF A SET AND THEIR SIZES}

For $x \in X$ set

$r(D, x)=\sup \{\|x-d\|: d \in D\}$

$r(D)=\inf \{r(D, x): x \in X\} \quad$ (radius of $D)$;

$r(D, D)=\inf \{r(D, x): x \in D\} \quad$ (self-radius of $D)$.

A point $c \in X$ such that $r(D, c)=r(D)$ is called a center of $\mathrm{D}$. Note that not always a center exists, but in finite dimensional case its existence is guaranteed. We have always $r(D) \leq r(D, D) \leq$ $\delta(D) \leq 2 r(D)$, and also, $r(D, D)=r(D)$ if $D$ is complete (see [1, Theorem 3.3]).

Clearly, we always have

(3) $\delta\left(D^{\prime}\right) \leq 2 r\left(D^{\prime}\right) \leq 2 r\left(D^{\prime}, D^{\prime}\right) \leq 2 \inf \left\{r\left(D^{\prime}, x\right): x \in D\right\} \leq 2 \delta(D) \leq 4 r(D)$.

Note that $r\left(D^{\prime}, D^{\prime}\right)<\inf \left\{r\left(D^{\prime}, x\right): x \in D\right\}$ in Example 5 (in Section 3). The following examples show that also (3) is sharp, since the equality $\delta\left(D^{\prime}\right)=4 r(D)$ is possible.

Example 1. In fact, our first example consists of three examples; the third one will be used further on.

A) Consider in $R^{2}$, with the max norm, the set $D=\{(x, 0): 0 \leq x \leq 1\}$. We have: $D=D^{c}$ and $D^{\prime}=\{(x, 0): 0 \leq x \leq 1,|y| \leq 1\}$, and so $\delta\left(D^{\prime}\right)=2$ and $r(D)=1 / 2$.

B) Consider the space $R^{3}$ with the sum (or $\ell_{1}$ ) norm. Let $D$ be the convex hull of $\{(1,1,0) ;(1,0,1) ;(0,1,1)\}$. We have: $\delta(D)=2$ and $r(D)=1((1,1,1)$ is the unique center of $D)$. Both points $(0,0,0)$ and $\left(\frac{4}{3}, \frac{4}{3}, \frac{4}{3}\right)$ belong to $D^{\prime}$, and their distance is 4 . Also, according to $\left(2^{\prime}\right), D$ has different completions.

C) $D$ is the set indicated in A), but in the space $R^{2}$ with the Euclidean norm. We have:

$D^{c}=B((1 / 2,-\sqrt{3} / 2), 1) \cap B((1 / 2, \sqrt{3} / 2), 1) ; D^{\prime}=B(O, 1) \cap B((1,0), 1)$.

For $d \in D$, set

$r^{\prime}(D, d)=\inf \{\|x-d\|: x \notin D\}$

$r^{\prime}(D)=\sup \left\{r^{\prime}(D, x): x \in D\right\} \quad$ (inner radius of $\left.D\right)$.

Note that if $D$ has empty interior, then $r^{\prime}(D)=0$.

Given $D, x \in D^{\prime}$ means $\|x-d\| \leq \delta(D)$ for all $d \in D$. So we have that 
$\left(P_{2}\right) \quad$ if $x \in D^{\prime}$, then $r(D, x) \leq \delta(D) \quad$ (and conversely).

If $D$ has interior points and $B\left(d_{o}, \alpha\right) \subset D\left(d_{0} \in D ; \alpha>0\right)$, then $\left\|x-d_{o}\right\| \leq \delta(D)-\alpha$ for all $x \in D^{\prime}$; so $\|x-y\| \leq\left\|x-d_{0}\right\|+\left\|d_{0}-y\right\| \leq 2(\delta(D)-\alpha)$ for $x, y \in D^{\prime}$. Therefore (3) can be improved to

$\left(3^{\prime}\right) \quad \delta\left(D^{\prime}\right) \leq 2 \delta(D)-2 r^{\prime}(D)$.

Moreover (with the same notations as above), $x \in D^{\prime} \backslash D$ implies $d_{o}^{\prime}=d_{o}+\alpha \frac{x-d_{o}}{\left\|x-d_{o}\right\|} \in D$ and $\left\|x-d_{o}^{\prime}\right\| \leq r(D, x)-2 \alpha$. Thus, denoting by $H(A, B)$ the Hausdorff distance between the sets $A$ and $B$, we have

$$
H\left(D, D^{\prime}\right) \leq \delta(D)-2 r^{\prime}(D) \leq 2\left(r(D)-r^{\prime}(D)\right) .
$$

The inequality $\left(3^{\prime}\right)$ is sharp; see Example $\left.1 \mathrm{~A}\right)$. Or also, use as $D$ the set denoted by $D^{\prime}$ in the same example (for which $r^{\prime}=1 / 2$ and $\delta=2$ ). The same holds for the inequalities in $\left(3^{\prime \prime}\right)$. Moreover, no better estimate is possible if we consider $D^{c}$ instead of $D^{\prime}$ : we give an example where $D$ has nonempty interior, its completion is unique and equalities hold in $\left(3^{\prime \prime}\right)$.

Example 2. Consider in $R^{2}$, with the max norm, the set $D=\{(x, y): 0 \leq x \leq 1,0 \leq y \leq x\}$. We have: $\delta(D)=1, r(D)=1 / 2, r^{\prime}(D)=1 / 4, D^{\prime}=D^{c}=\{(x, y): 0 \leq x \leq 1,0 \leq y \leq 1\}$ (this is the unique completion of $D)$, and $H\left(D, D^{c}\right)=H\left(D, D^{\prime}\right)=1 / 2$.

The inequality $H\left(D, D^{c}\right) \leq r(D)$ is not true in general (see Example 5 in Section 3), so $r(D)$ is not so useful in this context. Now we shall consider $r(D, D)$.

The following was proved in [1, Theorem 3.5]:

$\left(P_{3}\right)$ Let $D \subset B(x, r)$ (for some $x \in X$ and $r \in R$ ). Then $D$ has a completion contained in $B(x, r)$ if $r \leq \delta(D)$ (so $D^{c} \subset B(x, r)$ ).

Indeed, the last bound for $r$ was not indicated in 1, but the proof given there only works for $r \leq \delta(D)$. Example $4 \mathrm{~A}$ ) below shows that such bound is crucial.

Proposition 1. We always have

$H\left(D, D^{c}\right) \leq r(D, D)$.

Proof. If $r(D, D)=\delta(D)$, the result is contained in $\left(3^{\prime \prime}\right)$.

Otherwise, let $\varepsilon \in(0, \delta(D)-r(D, D)]$; take $d \in D$ such that $D \subset B(d, r(D, D)+\varepsilon) \subset$ $B(d, \delta(D))$. Then, according to $\left(P_{3}\right)$, also $D^{c} \subset B(d, r(D, D)+\varepsilon)$. Thus $x \in D^{c} \Longrightarrow\|x-d\| \leq$ $r(D, D)+\varepsilon$ with $d \in D$, and then $H\left(D, D^{c}\right) \leq r(D, D)+\varepsilon$. Since $\varepsilon>0$ is arbitrary, we obtain the thesis.

Note that the inequality $H\left(D, D^{\prime}\right) \leq r(D, D)$ is not true in general. Our next example shows this.

Example 3. Let, for $t \in[0,1 / 2], D_{t}=\{f \in C[-1,1]: 0 \leq f(x) \leq t(x+1)$ for $-1 \leq x \leq 0 ; 0 \leq$ $f(x) \leq 1$ for $0 \leq x \leq 1\}$. Among the completions, there are sets of functions satisfying, for $-1 \leq x \leq 0, f(x) \in[\alpha-1, \alpha], t \leq \alpha \leq 1$; or also $t(x+1)-1 \leq f(x) \leq t(x+1)$. We have: $r\left(D_{t}\right)=1 / 2, \delta\left(D_{t}\right)=1, r\left(D_{t}, D_{t}\right)=1-t, \delta\left(D_{t}^{\prime}\right)=2$, and $H\left(D_{t}, D_{t}^{\prime}\right)=1$.

The difference $\delta\left(D^{\prime}\right)-\delta(D)(\leq \delta(D))$ measures, in a sense, how different the completions of $D$ can be. We have 
Proposition 2. Let

$H(D)=\sup \left\{H\left(D_{1}, D_{2}\right): D_{1}, D_{2}\right.$ are completions of $\left.D\right\}$.

Then $\delta\left(D^{\prime}\right)-\delta(D) \leq H(D)$.

Moreover, equality holds if any complete set $C$ satisfies the following, slightly stronger condition (usually called "constant diameter", see [10, Section 3]):

(CD) $\quad r(C, x)=\delta(C)+\operatorname{dist}(x, C)$ for every $x \notin C$, where dist denotes the usual distance with respect to the norm under consideration.

Proof. We give the proof assuming that the values involved are attained; otherwise they can be arbitrarily well approximated, and the proof can be easily adapted (so the result is still true). Let $\delta\left(D^{\prime}\right)=\delta(D)+\alpha, \alpha>0$ (if $\alpha=0$, then $D^{\prime}$ is the unique completion of $D$, and there is nothing to prove). Take $x, y \in D^{\prime}$ such that $\|x-y\|=\delta\left(D^{\prime}\right)$; according to (2) there are two completions of $D$, say $D_{1}$ and $D_{2}$, such that $x \in D_{1}, y \in D_{2}$. Since $r\left(D_{1}, x\right) \leq \delta(D)$ (see $\left(P_{2}\right)$ ), to reach $y$ from $D_{1}$ we have to enlarge $D_{1}$ at least by $\alpha$. So the conclusion for the first part holds.

To prove the second part, let $H(D)=H\left(D_{1}, D_{2}\right)\left(D_{1}\right.$ and $D_{2}$ again being completions of $\left.D\right)$. Assume, for example that for every $\varepsilon>0$, there exists $x \in D_{2} \subset D^{\prime}$ such that $\|x-y\|>H(D)-\varepsilon$ for every $y \in D_{1}$ (otherwise, the role of $D_{1}$ and $D_{2}$ should be exchanged). Then (by using (CD) $r\left(D_{1}, x\right) \geq \delta\left(D_{1}\right)+H(D)-\varepsilon ; \quad$ so $\left(\delta\left(D_{1}\right)=\delta(D)\right)$ there exists $\bar{y} \in D_{1} \subset D^{\prime}$ such that $\|x-\bar{y}\|>\delta(D)+H(D)-2 \varepsilon$. This implies $\delta\left(D^{\prime}\right) \geq \delta(D)+H(D)$.

Remark 1 . We do not know if the assumption given for the second part of the previous proposition is necessary.

We always have $H(D) \leq \sup \left\{H\left(D_{1}, D\right): D_{1}\right.$ is a completion of $\left.D\right\}$. (Note that by the above Example $1 \mathrm{C})$, strict inequality is possible.)

In fact, let $\varepsilon>0$. According to Proposition 2 there are $D_{1}, D_{2}$ such that $\sup \left\{\operatorname{dist}\left(x, D_{2}\right): x \in\right.$ $\left.D_{1}\right\}>H(D)-\varepsilon$. Then $\sup \left\{\operatorname{dist}(x, D): x \in D_{1}\right\}>H(D)-\varepsilon$, so the conclusion.

Also, we have that $\inf \left\{H\left(D_{1}, D\right): D_{1}\right.$ is a completion of $\left.D\right\} \geq H\left(D, D^{c}\right)$. In general this is not an equality (see the same example quoted above).

Theorem 1. Let $D_{1}$ and $D_{2}$ be different complete sets. We have:

(a) if there is no inclusion between $D_{1}$ and $D_{2}$, then $D_{1} \cup D_{2}$ is not complete;

(b) if $\delta\left(D_{1}\right)=\delta\left(D_{2}\right)=\delta\left(D_{1} \cap D_{2}\right)$, then $D_{1} \cap D_{2}$ is not complete.

Proof. (a): Let $\delta\left(D_{1}\right)=d_{1} \leq d_{2}=\delta\left(D_{2}\right)$. We cannot have $\delta\left(D_{1} \cup D_{2}\right) \leq d_{2}$, since $\left(D_{1} \cup D_{2}\right)$ strictly contains $D_{i}, i=1,2$. Let $\delta\left(D_{1} \cup D_{2}\right)=d_{2}+\varepsilon, \varepsilon>0$.

Case 1: Let $D_{1} \cap D_{2} \neq \emptyset$.

Assume that $D_{1} \cup D_{2}$ is complete. There is a point $z$ which is at the same time in $\partial\left(D_{1} \cup D_{2}\right)$ and in $\partial\left(D_{1} \cap D_{2}\right)$. This implies $\sup \left\{\|z-y\|: y \in D_{1} \cup D_{2}\right\} \leq d_{2}$; but also (boundary points are endpoints of diameters in complete sets) $\sup \left\{\|z-y\|: y \in D_{1} \cup D_{2}\right\}=d_{2}+\varepsilon$. This contradiction shows that $D_{1} \cup D_{2}$ is not complete.

Case 2: $D_{1} \cap D_{2}=\emptyset$.

In this case $D_{1} \cup D_{2}$ is not convex. In fact, let $x \in D_{1}, y \in D_{2}$. The sets $[x, y] \cap D_{1}$ and $[x, y] \cap D_{2}$ are convex (and disjoint). Let $[x, y] \cap D_{1}=\left[x, x^{\prime}\right],[x, y] \cap D_{2}=\left[y^{\prime}, y\right]\left(x^{\prime} \neq y^{\prime}\right)$. So $\left(x^{\prime}, y^{\prime}\right)$ is not contained in $D_{1} \cup D_{2}$. 1,2 .

(b): It is enough to observe that $D_{1} \cap D_{2}$ is strictly contained in each of the two sets $D_{i}, i=$ 
Corollary 1. If $D$ has two different completions $D_{1}$ and $D_{2}$, then neither $D_{1} \cup D_{2}$ nor $D_{1} \cap D_{2}$ can be complete.

Proof. The first part follows from part (a) of the previous theorem. For the second part, note that $D_{1} \cap D_{2}$ contains $D$ (so $\delta\left(D_{1} \cap D_{2}\right)=\delta(D)$ ), and then apply part (b) of the previous theorem.

To prove our next result, we need the following lemma.

Lemma 1. Let $D$ be a set, and let $\alpha, \varepsilon>0$. Set

$$
A=\bigcap_{x \in D} B(x, \alpha) ; A_{\varepsilon}=\bigcap_{x \in D} B(x, \alpha+\varepsilon) .
$$

If $A \neq \emptyset$, then $\delta\left(A_{\varepsilon}\right) \geq \delta(A)+2 \varepsilon$.

Proof. Let $\delta(A)=d(d>0$ by $A \neq \emptyset)$. Given $\sigma>0$, take $y_{1}$ and $y_{2}$ in $A$ such that $\left\|y_{1}-y_{2}\right\| \geq d-\sigma$. On the line joining $y_{1}$ and $y_{2}$, take $y_{1}^{\prime}$ and $y_{2}^{\prime}$ so that $\left\|y_{1}-y_{1}^{\prime}\right\|=\varepsilon=\left\|y_{2}-y_{2}^{\prime}\right\|,\left\|y_{1}^{\prime}-y_{2}^{\prime}\right\|=$ $\left\|y_{1}-y_{2}\right\|+2 \varepsilon$. Since $y_{1} \in A$, we have $y_{1}^{\prime} \in A_{\varepsilon}$; similarly, $y_{2}^{\prime} \in A_{\varepsilon}$, so $\delta\left(A_{\varepsilon}\right) \geq d-\sigma+2 \varepsilon$. Since $\sigma>0$ is arbitrary, then the thesis follows.

Remark 2. In the above lemma, we can have $\delta\left(A_{\varepsilon}\right)>\delta(A)+2 \varepsilon$. For example, if we consider as $A$ the set denoted by $D^{\prime}$ in Example $1 \mathrm{C}$ ) and we take $\alpha=\varepsilon=1$, then $\delta(A)=\sqrt{3}, \delta\left(A_{\varepsilon}\right)=3 \sqrt{3}$.

Theorem 2. Let $A, B$ be sets such that $\delta(A) \neq \delta(B)$. Then $A^{c} \neq B^{c}$ and $A^{\prime} \neq B^{\prime}$.

Proof. If $\delta(A) \neq \delta(B)$, then $\delta\left(A^{c}\right) \neq \delta\left(B^{c}\right)$, so $A^{c} \neq B^{c}$.

For the second part, assume (by contradiction) that $A^{\prime}=B^{\prime}$. For example, let $\delta(A)=d, \delta(B)=$ $d+\varepsilon, \varepsilon \geq 0 ;$ then $\left(\right.$ see $\left.\left(P_{1}\right)\right)$

and

$$
\left.A^{c}=\bigcap\{B(x, d)): x \in A^{\prime}\right\}
$$

$B^{c}=\bigcap\left\{B(x, d+\varepsilon): x \in A^{\prime}\right\}$.

Therefore, according to Lemma 1, we have $d+\varepsilon=\delta(B)=\delta\left(B^{c}\right) \geq \delta\left(A^{c}\right)+2 \varepsilon$. This proves that $\varepsilon=0$, so $\delta(B)=\delta\left(B^{c}\right)=\delta\left(A^{c}\right)=\delta(A)$.

It is clear that, in general, the inclusion $A \subset B$ together with the equality $\delta(A)=\delta(B)$ implies $A^{c} \subset B^{c} ; B^{\prime} \subset A^{\prime}$.

Moreover, the inequality $\delta\left(B^{\prime}\right)<\delta\left(A^{\prime}\right)$ is possible: let $A$ be the set $D$ in Example $1 \mathrm{C}$ ) and $B=B(O, 1)$.

Under the same assumptions on $A$ and $B$, if $A$ has a unique completion (this means $A^{c}=A^{\prime}$ ), then $B$ has the same unique completion since $A^{c} \subset B^{c} \subset B^{\prime} \subset A^{\prime}$. So we have

$\left(P_{4}\right)$ If $A \subset B, \delta(A)=\delta(B)$, and $A$ has a unique completion $A^{\prime}$, then $A^{\prime}$ is also the unique completion of $B$.

But it is possible that $B$ has a unique completion (in particular, $B$ is complete), and $A \subset B$ has more completions.

Also we can have $\delta(A)<\delta(B)$ and $\delta\left(A^{\prime}\right)=\delta\left(B^{\prime}\right)$.

In general, the inclusion $A \subset B$ does not imply that any inclusion between $A^{c}$ and $B^{c}$ or between $A^{\prime}$ and $B^{\prime}$ holds (unless $A$ and $B$ have a unique completion). We give an example showing this. 
Example 4. Let $X$ be the Euclidean plane.

A) Let $A$ be the equilateral triangle determined by the points $(-1,0),(1,0),(0, \sqrt{3})$, and $B$ be the ball $B\left((0, a), \sqrt{a^{2}+1}\right), a \geq 1 / \sqrt{3}$. The boundary of $B$ contains the first two vertices of $A$, and we have: $A \subset B, \delta(A)<\delta(B)$, and $B^{c}=B$ is not contained in $A^{c}$. Moreover, for $a>\sqrt{3}$ the unique completion of $A$ (a Reuleaux triangle $T=A^{c}=A^{\prime}$ ) is not contained in $B=B^{c}=B^{\prime}$.

B) Let $A=\{(x, 0):|x| \leq 1 / 2\}$ and $B=B(0,1 / 2+\varepsilon)$ with $\varepsilon>0$ "small". Then there is no inclusion between $B^{\prime}=B$ and $A^{\prime}$.

We prove another result. For two different sets $A$ and $B$ it is possible to have $A^{c}=A^{\prime}=B^{\prime}=B^{c}$ (for example, let $B$ be the unique completion of an incomplete set $A$ ). In any case, the following fact is true.

Proposition 3. Given two sets $A$ and $B$, we have

$A^{c}=B^{c} \Leftrightarrow A^{\prime}=B^{\prime}$.

Proof. Let $A^{c}=B^{c}$. Then $A^{\prime}=\left(A^{c}\right)^{\prime}=\left(B^{c}\right)^{\prime}=B^{\prime}$.

Conversely, let $A^{\prime}=B^{\prime}$. Then, according to Theorem 2, we have $\delta(A)=\delta(B)=\delta$. So, by using $\left(P_{1}\right)$, we obtain: $A^{c}=\bigcap_{x \in A^{\prime}} B(x, \delta)=\bigcap_{x \in B^{\prime}} B(x, \delta)=B^{c}$.

\section{Completion of Sets, RADil, AND CENTERs}

Some of the results in this section have been indicated, for finite dimensional spaces, in $[\underline{6}$.

Every center of $D$ is contained in $D^{\prime}$ (see 1, Theorem 3.3]), but not necessarily in $D^{c}$, also when there is a unique center for $D$. In fact: for the set $D$ in Example $1 \mathrm{~B}),(1,1,1) \notin B(O, 2)$, where $2=\delta(D)$, and $D \subset B(O, 2)$; so also $D^{c} \subset B(O, 2)$.

It is clear that in general $D^{c}$ does not contain a completion of $D$.

Let $D \subset B(x, \delta(D))=B_{x}$. If $x \in D$, then $D^{\prime} \subset B_{x}$, and so all completions of $D$ are contained in $B_{x}$. But otherwise, in general, this is not true. For example, consider the set $D$ in Example 1 C) $\quad(\delta(D)=1)$ and $x=(1 / 2,1 / 2)$.

According to $\left(P_{3}\right), r(D)=r\left(D^{c}\right)$ (see [1, Corollary 3.6]) and $r(D)=\inf \{r(A): A$ is a completion of $D\}$.

Clearly, $r(D)$ is also a minimum in the above formula when $D$ is complete, but also in the following cases:

- $D$ has a unique completion $D^{c}$;

- $D$ has a center (see the discussion above).

But in some cases the infimum is not a minimum; see [1, Example 3.3]. Also (and as already said) there exist complete sets without centers (see [3, Example 1]).

Clearly, in general $r\left(D^{\prime}\right) \geq r(D)$ (inequality is strict in Example $\left.1 \mathrm{C}\right)$ ). According to (3), $r\left(D^{\prime}\right) \leq 2 r(D)$, and this estimate is sharp (see Example $\left.1 \mathrm{~A}\right)$ ).

Note that a set can have a unique center and different completions (see Example $1 \mathrm{C}$ )).

In general, different completions of a given set can have different radii; also, we can have different completions with the same radius. The range of $r\left(D_{m}\right), D_{m}$ denoting completions of $D$, can be the whole interval $[r(D), 2 r(D)]$. To see this, look at Example 3, with $t=0$. In fact, we have: $D$ has completions with the same radius $r=r(D) \quad(\alpha=1)$ and completions with radius $r>r(D)$ $(r=1$ for $\alpha=0)$.

The same example, with $\alpha=0$, also shows that complete sets can have more centers. 
Our next result extends [6, Corollary 3].

Theorem 3. The sets $D$ and $D^{c}$ have the same centers and the same completions.

Proof. Recall that $D$ and $D^{c}$ have the same diameter and the same radius.

Let $r\left(D^{c}\right)=r(D)=r$. If $c$ is a center of $D^{c}$ (i.e., $D^{c} \subset B(c, r)$ ), then it is also a center of $D$. Conversely, if $c$ is a center of $D$ (i.e., $D \subset B(c, r)$ ), then by $\left(P_{3}\right) D^{c} \subset B(c, r)$, and so $c$ is also a center of $D^{c}$.

For the second part: a completion of $D$ must contain $D^{c}$ (see (1)), so it is also a completion of $D^{c}$. The converse is clear.

Now we compare the completions of $D^{\prime}$ with those of $D$.

If $D$ has more completions, then $\delta\left(D^{\prime}\right)>\delta(D)$, so $D$ and $D^{\prime}$ have different completions. Moreover (see [1, Theorem 3.8]), we have $\delta\left(\left(D^{\prime}\right)^{\prime}\right)>\delta\left(D^{\prime}\right)$ ), so also $D^{\prime}$ has more completions.

Let $D$ have a unique completion $D^{\prime}$. Then $r(D)=r\left(D^{\prime}\right)=r\left(D^{\prime}, D^{\prime}\right)$. But we can have $r(D, D)>r(D)$; see the next example. Note that if, moreover, $D$ has no center, then (since $\left.r(D)=r\left(D^{\prime}\right)\right)$ the same is true for all sets $A$ satisfying $D \subset A \subset D^{\prime}$.

Example 5. Let $D=\{f \in C[0,1]: f(0)=0 ; 0 \leq f(x) \leq 1\}$. Its unique completion is $D^{\prime}=B(\bar{f}, 1 / 2)$, where $\bar{f}$ is the constant function $1 / 2$ (we have $r(D)=r\left(D^{\prime}\right)=r\left(D^{\prime}, D^{\prime}\right)=$ $\left.1 / 2 ; r(D, D)=1=\delta(D)=\delta\left(D^{\prime}\right)\right)$.

We noticed that, in case of a unique completion, $r(D)=r\left(D^{c}\right)=r\left(D^{\prime}\right)$. The converse is not true. Namely, the condition $r(D)=r\left(D^{\prime}\right)$ does not imply $\delta(D)=\delta\left(D^{\prime}\right)$, as our next example shows (see [1, Theorem 3.7, $(k) \nRightarrow(h)])$.

Example 6. Consider the space $c_{o}$ of all sequences converging to 0, with the max norm. Let $D=\left\{x=\left(x_{1}, x_{2}, \ldots, x_{n}, \ldots\right): x_{1}=0 ; 0 \leq x_{i} \leq 1\right.$ for $\left.i \geq 2\right\}(\delta(D)=1)$. The set $D$ has different completions $\left(\delta\left(D^{\prime}\right)=2\right)$; but we have $r(D)=r\left(D^{\prime}\right)=r(D, D)=1$.

Let $A \subset B$ and $\delta(A)=\delta(B)$. Then $A^{c} \subset B^{c}$, and so $r\left(A^{c}\right) \leq r\left(B^{c}\right)$. In general, the last inequality is not an equality. For example, consider in the Euclidean plane $A=\{(x, 0):|x| \leq 1 / 2\}$ and $B$ an equilateral triangle based on $A$.

Also, under the same assumptions we have $A^{\prime} \supset B^{\prime}$. So $r\left(A^{\prime}\right) \geq r\left(B^{\prime}\right)$, and in general the last inequality is not an equality, as the same example above shows.

If $A \subset B$ and $\delta(A)<\delta(B)$, then, concerning $r\left(A^{\prime}\right)$ and $r\left(B^{\prime}\right)$, all cases are possible $(<,=,>)$. In fact, the possibility $r\left(A^{\prime}\right)<r\left(B^{\prime}\right)$ is trivial; equality is possible according to Example 6 above (take $\left.A=D, B=D^{\prime}\right)$. Concerning $r\left(A^{\prime}\right)>r\left(B^{\prime}\right)$, see Example $4 \mathrm{~B}$ ).

We present a result indicated in [1] (see Remark to Corollary 3.4 there) and an immediate consequence of it. We limit ourselves to the consideration of sets where centers exist, but the general case could be treated in a similar way, giving estimates for the set of approximate centers of $D$.

For a set $D$, we denote by $C_{D}$ the set of its centers.

Proposition 4. For every set $D$ the following inequality is true:

$\delta\left(C_{D}\right) \leq 2 r(D)-2 \delta(D)+\delta\left(D^{\prime}\right)$.

In particular, if $D$ has a unique completion, then

$\delta\left(C_{D}\right) \leq 2 r(D)-\delta(D) \leq \delta(D)$. 
Remark 3. The inequality $\delta\left(C_{D}\right) \leq 2 r(D)-\delta(D)$ is not true in general. In fact, in Example 6 we have $\delta(D)=r(D)=1, \delta\left(C_{D}\right)=\delta\left(D^{\prime}\right)=2$. The first inequality is sharp; see Example $\left.1 \mathrm{~A}\right)$.

Concerning the second statement in Proposition 4, the first inequality is sharp; for example, it becomes an equality for a ball. Of course, also the second is sharp; see Example 6 .

The second part also implies the following fact: if $\delta\left(C_{D}\right)>\delta(D)$, then $D$ has more completions.

\section{Completions and Minimality}

The sets we are considering will always be assumed to be bounded, closed and convex with diameter $>0$.

Let $C$ be a complete set of diameter $d$.

Consider a set $D \subset C$ with $\delta(D)=d$ (in particular, $D=C$ ). Then set

$T(D, C)=\{A \subset D: C$ is a completion of $A\}$.

Also, let $C$ be the unique completion of $D\left(D^{c}=D^{\prime}=C\right)$; set

$U(D, C)=\{A \subset D: C$ is the unique completion of $A\}=\left\{A \subset D: A^{c}=A^{\prime}=C\right\}$.

We shall discuss minimality for completions, or for unique completions.

If $\mathrm{T}(\mathrm{D}, \mathrm{C})$ (resp. $\mathrm{U}(\mathrm{D}, \mathrm{C})$ ) is the singleton $\{D\}$, then we say that $D$ is $(\mathrm{mC})$ (resp.: $(\mathrm{muC})$ ). Otherwise, if $D$ is not $(\mathrm{mC})$, or not $(\mathrm{muC})$, then an application of Zorn's lemma (every chain has a lower bound) shows that $D$ contains minimal subsets with that property. In other words, there are subsets of $D$ which are minimal in the sense of inclusion, that still have $C$ as completion (resp.: as unique completion).

Note that " $D$ is $(\mathrm{mC})$ " means the following: $D \subset C, \delta(D)=d, \delta(S)<d$ for every proper subset $S$ of $D$. So the condition " $D$ is $(\mathrm{mC})$ " is equivalent to: the maximal width of $S$ is smaller than $d$ for every proper subset $S$ of $D$ (see [4, Proposition 4]). Apparently, a related notion (less tractable) is the following: the minimal width of a convex body $D$ is smaller for every proper subset $S$ of $D$ (i.e., $D$ is reduced; see, for example, [5]). Reducedness does not imply (mC) (look at the equilateral triangle in the Euclidean plane); a segment is $(\mathrm{mC})$ but it is not reduced (it is not a body).

We shall discuss the following questions.

(Q1) Which sets $D$ are (mC)?

(Q2) Which sets $D$ are (muC)?

The answer to (Q1), at least in finite dimensional spaces, is trivial. The answer to (Q2) seems to be difficult.

Proposition 5. If $X$ is a finite dimensional normed space, then, given a complete set $C$ of diameter $d, D \subset C$ is $(\mathrm{mC})$ if and only if it is a segment of diameter (length) $d$ contained in $C$.

Proof. If $D$ is a segment of diameter (length) $d$, then any proper subset of $D$ has diameter $<d$, so $C$ is not a completion of it.

Assume that $D$ is $(\mathrm{mC})$ (i.e., $\delta(D)=d$ ). Take a diametral pair $x, y$ of $D$. Then the segment $[x, y]$ is $(\mathrm{mC})$. If $D$ is not a segment, then $[x, y]$ is a proper subset of $D$, so $D$ cannot be $(\mathrm{mC})$.

Remark 4. Proposition 5 is not true if $X$ is infinite dimensional. In fact, in this case we can consider a set $D$ without a diametral pair (see 14]); this means that if $\delta(D)=d$, then for every pair $x, y \in D$ we have $\|x-y\|<d$. Now let $C$ be a completion of $D$. There is a minimal subset $A$ of $D$ having $C$ as a completion. But $A$ cannot be a segment, since every segment contained in $D$ has diameter (length) $<d$. 
Note that a segment can have a unique completion or more completions, as the next example shows.

Example 7. Let $X$ be the plane with the max norm. Let $C=\{(x, y): 0 \leq x \leq 1 ; 0 \leq y \leq$ $1\}, D_{1}=\{(x, y): 0 \leq x \leq 1 ; y=0\}, D_{2}=\{(x, y): 0 \leq x \leq 1 ; x=y\}, D_{3}=\{(x, y): 0 \leq x \leq$ $1 ; y=1-x\}$. Then $D_{1}$ has more completions, while $D_{2}$ and $D_{3}$ have $C$ as unique completion.

Now we discuss $\left(Q_{2}\right)$. If $D$ has the unique completion $C$, then $\delta(D)=d=\delta(C)$, and moreover: $D^{c}=D^{\prime}=C$. This means that $\left(\right.$ see $\left.\left(P_{1}\right)\right)$ :

$D^{c}=\bigcap_{x \in C} B(x, d)=\bigcap_{x \in D} B(x, d)=D^{\prime}$,

while if $S$ is a proper subset of $D$, of diameter $d$, then $\delta\left(S^{\prime}\right)=\delta\left(\bigcap_{x \in S} B(x, d)\right)>d$.

Note that in this case, since $D^{c}=D^{\prime}=C$, then $S^{c}$ should be strictly contained in $C$ (according to Proposition 3).

Segments are (muC) only if they have a unique completion $C$ (see Example 7). We now give an example of a (muC) set which is not a segment.

Example 8. Let $X$ be the plane with the max norm. Let $C=\{(x, y): 0 \leq x \leq 1 ; 0 \leq y \leq$ $1\}, D_{4}=\{(x, y): 0 \leq x \leq 1 ; x / 2 \leq y \leq 1-x / 2\}$. Then $D_{4}$ has $C$ as unique completion, while every proper subset of $D_{4}$ has more completions. The same is true if we consider an equilateral triangle in the Euclidean norm.

Minimal elements are not unique in general. In Example 7, both $D_{2}$ and $D_{3}$ are (muC) (for $C$ as defined there); in that example, $C$ is the unique set with diameter 1 , containing both $D_{2}$ and $D_{3}$.

Note that if $M_{1}$ and $M_{2}$ are two different sets being $(\mathrm{muC})$, then it is clear that $\delta\left(M_{1} \cap M_{2}\right)<$ $\delta(C)$.

In Example 8, $D_{4}$ is not complete (its unique completion is $C$ ); each segment in the boundary of $D_{4}$ is $(\mathrm{mC})$.

\section{REFERENCES}

[1] M. Baronti and P.L. Papini, Diameters, centers and diametrically maximal sets, Rend. Circolo Mat. Palermo Suppl. (II) 38 (1995), 11-24.

[2] F. Bavaud, Adjoint transform, overconvexity and sets of constant width, Trans. Amer. Math. Soc. 333 (1992), 315-324.

[3] L. Caspani and P.L. Papini, Complete sets, radii, and inner radii, Beitr. Algebra Geom. 52 (2011), 163-170.

[4] L. Caspani and P.L. Papini, On constant width sets in Hilbert spaces and around, J. Convex Anal., to appear.

[5] M. Lassak and H. Martini, Reduced convex bodies in Euclidean space - a survey, Expos. Math. 29 (2011), 204-219.

[6] P. Martín, H. Martini and M. Spirova, Chebyshev sets and ball operators, submitted.

[7] H. Martini and M. Spirova, On the circular hull property in normed planes, Acta Math. Hungar. 125 (2009), $275-285$.

[8] H. Martini, Ch. Richter, M. Spirova, Intersections of balls and sets of constant width in finite dimensional normed spaces, Mathematika, 13 pp., to appear.

[9] J.P. Moreno, Porosity and unique completion in strictly convex spaces, Math. Z. 267 (2011), 173-184.

[10] J.P. Moreno, P.L. Papini and R.R. Phelps, New families of convex sets related to diametral maximality, J. Convex. Anal. 13 (2006), 823-837.

[11] J.P. Moreno and R. Schneider, Local Lipschitz continuity of the diametric completion mapping, Houston J. Math. 38 (2012), 1207-1223.

[12] E.S. Polovinkin, On the construction of bodies of constant width containing a given set, Proc. Steklov Inst. Math. (2010) suppl. 2, S247-S257.

[13] E.S. Polovinkin and S.V. Sidenko, The completion of sets to bodies of constant width (Russian), Uch. Zap. Kazan. Gos. Univ., ser. Fiz.-Mat. Nauki 148 No. 2 (2006), 132-143. 
[14] L. Veselý, Any infinite-dimensional Banach space contains a convex body without diametral points for its Minkowski gauge, Boll. Un. Mat. Ital. A (7) 8 (1994), 95-99.

Authors' addresses:

Horst Martini, Fakultät für Mathematik, TU Chemnitz, D-09107 Chemnitz, Germany. e-mail: horst.martini@mathematik.tu-chemnitz.de

Pier Luigi Papini, Via Martucci 19, 40136 Bologna, Italy. e-mail: plpapini@libero.it

Margarita Spirova, Fakultät für Mathematik, TU Chemnitz, D-09107 Chemnitz, Germany. e-mail: margarita.spirova@mathematik.tu-chemnitz.de 